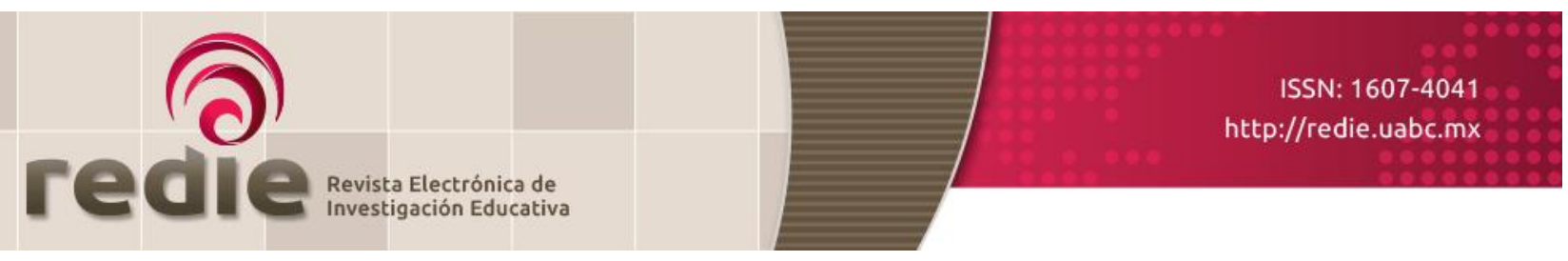

Vol. 20, Núm. 4, 2018

\title{
Estudio exploratorio de las emociones en la cotidianidad de las clases de Matemáticas
}

\section{Exploratory Study of Everyday Emotions in Mathematics Classes}

\author{
Josefa Perdomo Díaz (*) pepiperdomo@gmail.com \\ Andrés Fernández Vergara $\left(^{*}\right)$ andresefernandezv@gmail.com
}

(*) Universidad de Chile

(Recibido: 15 de diciembre de 2016; Aceptado para su publicación: 2 de marzo de 2017)

Cómo citar: Perdomo, J. y Fernández, A. (2018). Estudio exploratorio de las emociones en la cotidianidad de las clases de matemáticas. Revista Electrónica de Investigación Educativa, 20(4), 133-139. https://doi.ora/10.24320/redie.2018.20.4.1748

\section{Resumen}

Se presenta un estudio exploratorio cuyo objetivo es analizar el mapa emocional de un grupo de estudiantes chilenos de 4o. de Primaria durante la realización de actividades de la unidad "Medición: unidades de longitud, perímetro y área", estudiar diferencias de género e indagar la existencia de patrones emocionales. Se utiliza una perspectiva situada, que considera las emociones como parte de los afectos locales, de corta duración y ligadas a condiciones específicas. Los métodos empleados fueron el análisis de frecuencias, el test $\chi^{2}$ y el test no paramétrico de Kruskal-Wallis, y un análisis de clústeres. El mapa global muestra un $45 \%$ de emociones positivas, frente a un $18.3 \%$ de emociones negativas. Los hombres presentan mayor promedio de respuestas en blanco que las mujeres y se muestran menos preocupados. Por último, la clusterización permitió definir 5 patrones emocionales.

Palabras clave: Emociones, afectividad, matemáticas, educación básica, género.

\section{Abstract}

This paper presents an exploratory study that seeks to analyze the emotional map of a group of $4^{\text {th }}$-grade elementary students from Chile during activities in the unit "Measurement: units of length, perimeter and area", study gender differences, and explore the existence of emotional patterns. The study uses a situated perspective that considers emotions to be part of local affects, short-lived and linked to specific conditions. The methods employed are a frequency analysis, a $\chi^{2}$ test, a non-parametric Kruskal-Wallis test, and a cluster analysis. Globally, the map shows $45 \%$ positive emotions and $18.3 \%$ negative emotions. Men exhibit a higher average of blank responses than women, and appear less concerned. Lastly, clustering made it possible to define five emotional patterns. 


\section{Introducción}

Cada vez es mayor el reconocimiento de que el dominio afectivo juega un rol importante en el aprendizaje de cualquier disciplina. Existen resultados de investigaciones sobre la interacción entre las emociones y funciones cognitivas, como acceder a contenido mental, usar información o aplicar estrategias de resolución de problemas (Mandler, 1989), su influencia en la toma de decisiones y en las acciones que realiza un individuo (Ibañez, 2002; Schoenfeld, 1998), así como de su contribución a la formación de actitudes, creencias y motivación (Hannula, 2012; McLeod, 1992). Estudios más recientes muestran relaciones entre las emociones y los logros de aprendizaje (Holm, Hannula y Björn, 2016; Pekrun, Goetz, Frenzel, Barchfeld y Perry, 2011). A pesar del reciente aumento de investigaciones, éste sigue siendo el subdominio afectivo menos estudiado, pues los autores de trabajos en este campo se encuentran con dos dificultades: la ambigüedad del término "emoción" y la complejidad de acceder a las emociones de los individuos debido a su corta duración y su fuerte carácter personal y cultural (Hannula, 2012; Pekrun, 2005).

Los primeros trabajos en esta área se centraron en el estudio de la ansiedad matemática (McLeod, 1992), tema que continúa vigente en la actualidad, al que se le han sumado otros estados, como placer, orgullo, enfado, vergüenza, desesperanza o aburrimiento (Frenzel, Pekrun, y Goetz, 2007a; 2007b). Una parte importante de estas investigaciones se ha realizado en el contexto de situaciones académicas generales, sin referirse a actividades concretas en un momento determinado. Por ejemplo, se pregunta a los estudiantes por sus emociones en relación con las clases de Matemáticas, la realización de un examen o estudiar, sin especificar un momento o situación (Holm et al., 2016; Martínez-Sierra y García, 2014). La mayoría de los trabajos en un contexto específico de Matemáticas se han centrado en la resolución de problemas (DeBellis y Goldin, 2006; Op't Eynde, De Corte y Verschaffel, 2006). En cuanto a los participantes, a nivel internacional, la mayor parte de las investigaciones sobre emociones y el aprendizaje de las Matemáticas se han realizado con estudiantes de 14 años o más, salvo algunas excepciones donde los sujetos de estudio han sido alumnos de corta edad (DeBellis y Goldin, 2006; Frenzel et al., 2007a), profesores o estudiantes para profesor (Ibañez, 2002).

Esto muestra un amplio camino por recorrer hacia la comprensión de las emociones en la enseñanza y el aprendizaje de las Matemáticas, su estructura y su rol en el desempeño de los estudiantes. En este sentido, Hannula, Pantziara, Waege y Schlöglmann (2010), a partir de la revisión de los trabajos discutidos en el IV Congreso de la Sociedad Europea para la Investigación en Educación Matemática (CERME, por sus siglas en inglés), señalan la necesidad de ampliar el conocimiento que se tiene sobre las emociones en experiencias matemáticas rutinarias. El trabajo que se presenta en este artículo corresponde a esa línea de investigación. Se estudian las emociones que un grupo de estudiantes chilenos de 40. de Primaria (910 años) indican durante la realización de las actividades de uno de los capítulos del libro de texto que usan a diario en sus clases de matemáticas (Medición: unidades de longitud, perímetro y área). Las preguntas que guiaron la investigación fueron: ¿Con qué frecuencia los estudiantes indican las emociones: aburrido, desafiado, enfadado, feliz, indiferente y preocupado durante su trabajo en clase resolviendo actividades de medición de longitudes, perímetros y áreas? ¿Hay diferencia de género en las emociones señaladas por los estudiantes? ¿Pueden distinguirse patrones entre los perfiles emocionales de los estudiantes? Con estas preguntas se analizaron las emociones en un contexto situado en la cotidianidad de la sala de clase, tomando en cuenta la gama completa de actividades que el docente utiliza para la enseñanza de una unidad de contenido.

\subsection{Las emociones académicas}

En educación Matemática, el dominio afectivo se ha caracterizado por tres elementos: emociones, actitudes y creencias (McLeod, 1992), a los que se han incorporado componentes como el sistema de valores (DeBellis y Goldin, 2006) o la motivación (Hannula, 2012). En este esquema general, Goldin (2000) distingue entre afectos locales (sentimientos que cambian rápidamente) y globales, que pueden ser considerados como el resultado de haber transitado por determinados estados afectivos locales y tienen un carácter más estable. Goldin, además, distingue entre el plano de lo que sentimos y lo que expresamos, que sería más consciente que el primero y de carácter más cognitivo. Las etiquetas que 
utilizamos para expresar lo que sentimos serían "герresentaciones esquemáticas" del sentir, que llevan asociado un proceso de valoración por parte del individuo (Op't Eynde et al., 2006). Es decir, palabras como, por ejemplo, "feliz", "ansioso", "alegre" o "preocupado", son un nombre, una etiqueta que en un momento determinado se da a un sentimiento, de esta forma, el sentir queda representado por esa palabra.

En esta investigación se consideró que una emoción, como señala McLeod (1992), es una respuesta afectiva, caracterizada por ser visceral, intensa y de corta duración. Respuestas localizadas en un momento determinado, asociadas a condiciones específicas, lo que las sitúa en el campo de los afectos locales. Bajo este paradigma, comprender el rol de las emociones en el aprendizaje de las Matemáticas implica estudiar la naturaleza de esta variable afectiva en el día a día de la clase, lo que llevó a plantear la investigación desde una perspectiva situada (Volet y Järvelä, 2001), la cual considera como variable de estudio a la persona en su contexto.

A las emociones que tienen lugar en contextos formales, como colegios o universidades, Pekrun, Goetz, Titz y Perry (2002) las denominan "emociones académicas". Estos autores, en un programa de investigación que engloba resultados de 5 estudios cualitativos y 10 cuantitativos (3 longitudinales y 7 transversales) encontraron que las emociones académicas que los estudiantes manifiestan con mayor frecuencia son: ansiedad, placer, esperanza, orgullo, alivio, enfado, aburrimiento y vergüenza. La ansiedad sobresale por encima del resto, siendo también la más analizada en investigaciones de diferentes áreas (educación, psicología, sociología y neurociencia). Los resultados de estas investigaciones hacen referencia a contextos académicos generales, entre los que se incluyen las matemáticas; sin embargo, los resultados no son específicos de esta disciplina.

En Matemáticas, algunas de las experiencias emocionales que se han identificado son alegría o angustia frente a la realización de un examen, o temor, alivio, interés, aburrimiento, orgullo o autorreproche durante las clases (Martínez-Sierra y García, 2014). En el contexto particular de la resolución de problemas se han identificado otros estados, como curiosidad, desconcierto, estímulo, entusiasmo, frustración, desesperanza (Goldin, 2000; Op't Eynde et al., 2006) o satisfacción y desilusión (Martínez-Sierra y García, 2014). En cuanto a las diferencias de género, se han realizado investigaciones en contextos específicos. Por ejemplo, Frenzel et al. (2007a) muestran que, en Alemania, las alumnas de 5o. de primaria tienen un perfil emocional más negativo que los hombres, manifestando con mayor frecuencia emociones como ansiedad, desesperanza y vergüenza. En otro estudio realizado en Alemania, con estudiantes de diferentes grados académicos (de 5o. a 10o.), Goetz, Frenzel, Hall y Pekrun (2008) encontraron que los hombres superan a las mujeres a la hora de señalar que disfrutan de las Matemáticas, algo que también se ha observado en estudiantes de 14-15 años (grado 8o.) de Finlandia (Holm et al., 2016).

\section{Método}

Se realizó un estudio exploratorio planteado desde una perspectiva situada, al cual considera al individuo (los estudiantes) en su contexto (Volet y Järvelä, 2001). Los datos provienen de una muestra intencional de estudiantes de 4o. de Primaria de un colegio particular subvencionado de Santiago de Chile, quienes participaron de forma voluntaria. Se eligió esta muestra por las facilidades que la directora del colegio y la profesora del curso brindaron para acceder a ellos; de los 38 matriculados, se recogieron datos de 28 estudiantes (tabla I). Los restantes se excluyeron porque no asistían a clase o recibían atención especial y realizaban actividades diferentes al resto, o no indicaron alguna emoción en ninguna de las tareas.

Tabla I. Distribución de los participantes por género y edad

\begin{tabular}{lccc}
\hline & 9 años & 10 años & Total \\
\hline Hombre & 5 & 5 & 10 \\
Mujer & 6 & 12 & 18 \\
\hline Total & 11 & 17 & 28 \\
\hline
\end{tabular}


La variable estudiada fueron las emociones que los participantes declararon en las actividades de la unidad de contenido "Medición: unidades de longitud, perímetro y área". Se diseñó para ello un instrumento de autorreporte, tomando el libro de texto de los estudiantes y estampando un timbre (sello) con un listado de emociones en cada una de las 177 actividades del capítulo (ver figura 1). Рara configurar el listado se tomó en cuenta la edad de los participantes, las condiciones de recogida de los datos y resultados de investigaciones relacionadas.

El instrumento debía ser fácil y rápido de utilizar para que los estudiantes indicaran lo que habían sentido en cada una de las 177 actividades en las que trabajarían. Por tanto, el número de emociones era un factor relevante, ya que un listado muy amplio podía generar mayor confusión a la hora de seleccionar una opción, ralentizando el tiempo de respuesta. La edad de los estudiantes influyó en la elección de las alternativas, que se incluyeron puesto que debían ser estados fácilmente reconocibles por los participantes. Por último, analizando las investigaciones en situaciones académicas generales (Holm et al., 2016; Pekrun et al., 2002; 2011) y en el contexto de la resolución de problemas matemáticos (Goldin, 2000; Op't Eynde et al., 2006) se observaron coincidencias en tres opciones que se incorporaron al instrumento: aburrido, feliz y enfadado. Otros sentimientos analizados en esas investigaciones como, angustia, ansiedad, temor o frustración, fueron considerados, optando por incorporar la opción preocupado. Por último, se incluyó una alternativa neutra (indiferente) y otra muy utilizada en el contexto chileno, en relación con la resolución de problemas en matemáticas (desafiado).

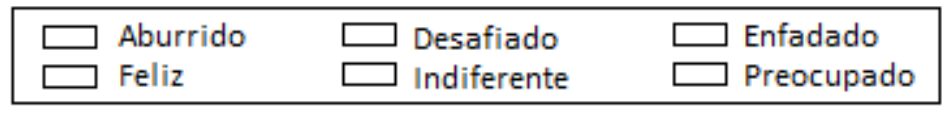

Figura 1. Imagen estampada en cada actividad de la unidad de contenido

El primer día de trabajo se mostraron los timbres a los estudiantes y se les explicó que debían marcar cómo se habían sentido cada vez que hicieran una actividad. Para el análisis se descartaron las 40 primeras actividades -consideradas como pilotaje del instrumento, lo que permitió que los alumnos se acostumbraran a señalar la emoción y aclararan las dudas que les surgían.

Una vez que la profesora del curso y los estudiantes terminaron su trabajo en esta unidad de contenido, se recogieron todos los libros de texto y se trasladó la información a una planilla Excel, asignando un código numérico a cada emoción. Se incluyó un código para las respuestas en blanco que en esta investigación son consideradas como una emoción más, ya que podrían estar indicando que la emoción que sentía el estudiante no se encontraba en el listado presentado.

En total se analizaron 3,836 datos, que corresponden a las emociones autorreportadas por 28 estudiantes en 137 actividades, incluyendo las respuestas en blanco.

\subsection{Análisis de los datos}

Los datos se analizaron con los paquetes estadísticos Stata y SPSS. La elección de los métodos se hizo según la naturaleza de las variables a estudiar, todas categóricas. En primer lugar se realizó un análisis de la frecuencia de cada uno de los valores de la variable Emoción, lo que permite mostrar un mapa emocional general de los participantes durante su trabajo en las actividades de medición. Para testear la hipótesis de independencia entre las variables Género y Emoción se aplicó el test $\chi^{2}$ de Pearson y el test no paramétrico de Kruskal-Wallis.

Enseguida se definió el perfil emocional de cada estudiante como una cadena de 137 caracteres, donde el caracter $i$ corresponde al código asignado a la emoción que el estudiante declaró en la actividad i-ésima. Por ejemplo, la cadena que comienza por 33066625 ... corresponde al perfil emocional de un alumno que declaró sentirse Aburrido en las dos primeras actividades, no indicó emoción en la tercera, indicó Feliz en las tres siguientes, Preocupado en la siguiente, luego Indiferente y así hasta completar 137 caracteres. Consideramos que dos estudiantes presentan un perfil emocional idéntico si sus respuestas coinciden en 
los 137 pares (actividad, emoción), es decir, si declaran las mismas emociones en las mismas actividades.

Para explorar la existencia de patrones emocionales se realizó un análisis de clúster entre los perfiles emocionales de los estudiantes, usando la distancia Hamming (1950), que cuenta la cantidad de caracteres que difieren entre dos cadenas de caracteres, y el método jerárquico de aglomeración de Ward (citado en Buehl y Alexander, 2005), que la literatura especializada destaca por su efectividad para descubrir estructuras de datos latentes (Atlas y Overall, 1994). El proceso de clusterización se representó gráficamente mediante un dendrograma que muestra en el eje horizontal a los 28 estudiantes y en el eje vertical el nivel de similitud de sus respuestas (ver figura 2). Esta representación resulta muy útil para agrupar a los estudiantes según sus respuestas ya que, elegido un nivel de similitud, el dendrograma indica cuántos grupos de estudiantes se generan y qué estudiantes forman parte de cada grupo. La decisión del número de patrones emocionales identificados se tomó conjugando la información obtenida en el dendrograma, los mapas de clústers y las tablas de promedios de cada emoción para cada grupo (ver Apéndice).

\section{Resultados}

\subsection{Mapa emocional del 40. básico}

El análisis de frecuencias de la variable Emoción muestra mayor porcentaje de emociones positivas (felizy desafiado) que negativas (enfadado, preocupado y aburrido), $45 \%$ y $18.3 \%$ respectivamente. Las opciones con mayor porcentaje fueron feliz (30.7\%), aburrido (11.6\%) y desafiado (9.8\%). Y las que menor porcentaje de respuestas obtuvieron son preocupado (5.1\%), enfadado (1.6\%) e indiferente (0.7\%). Destaca el alto porcentaje de respuestas en blanco, $40.6 \%$, que podría significar que los estudiantes no encontraron la emoción que sintieron en el listado proporcionado.

\subsection{Género}

El test de Pearson aplicado a la tabla de contingencia formada con las variables Emociones y Género indica que existe relación entre el género de los estudiantes y las emociones que declaran, $\chi^{2}(6$, $\mathrm{N}=3836$ ) $124.96, p<0.001$. El test no paramétrico de Kruskal-Wallis (tabla II) indica que hay diferencias significativas para las respuestas en blanco, enfadado, preocupado y feliz. Los hombres presentan mayor promedio de respuestas en blanco que las mujeres $(M=0.44, S D=0.29$ y $M=0.39, S D=0.24$, respectivamente) y se muestran menos preocupados ( $M=0.01, S D=0.03$ y $M=0.07, S D=0.09$, respectivamente). Los promedios para la opción feliz son iguales en ambos grupos ( $M=0.31)$, con SD=0.24 y $\mathrm{SD}=0.28$ en el caso de las mujeres y los hombres, respectivamente. Los resultados son difícilmente interpretables para enfadado por el escaso número de veces que los estudiantes declaran estas emociones (1.6\%).

Tabla II. Test de Kruskal-Wallis para las variables Emoción y Género

\begin{tabular}{l|c|c|c|c|c|c|c}
\hline & En Blanco & Enfadado & Preocupado & Aburrido & Indiferente & Feliz & Desafiado \\
\hline$\chi^{2}(6, \mathrm{~N}=3836)$ & 13.778 & 29.431 & 434.454 & 0.326 & 0.000 & 50.954 & 1.304 \\
\hline$p$ & 0.000 & 0.000 & 0.000 & 0.568 & 1.000 & 0.000 & 0.253 \\
\hline
\end{tabular}

\subsection{Patrones emocionales}

Usando la distancia Hamming para medir la separación entre dos perfiles y el método de Ward para formar los clústeres, se obtuvo el dendrograma de la figura 2, en el que puede verse, por ejemplo, que los perfiles de los estudiantes 22 y 28 son muy parecidos entre sí, así como los de los alumnos 1 y 6, o 7 y 23. 


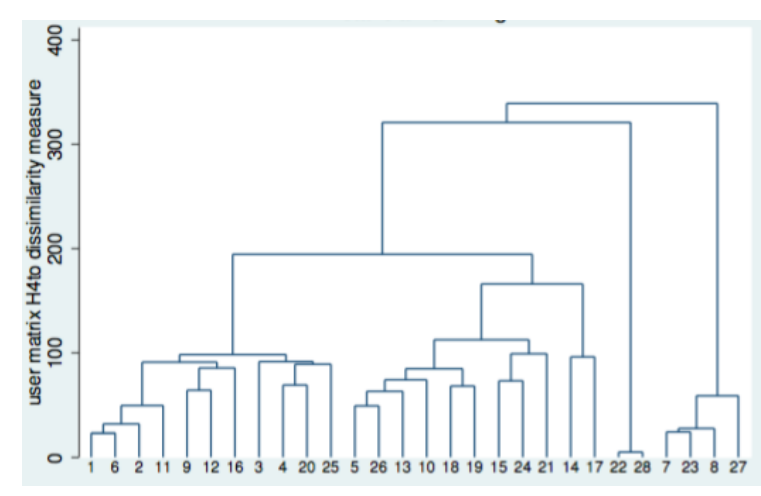

Figura 2. Dendrograma de las distancias Hamming entre los 28 estudiantes

La estructura del dendograma lleva a plantear la posibilidad de considerar 4, 5 o 6 clústeres. Рara tomar una decisión sobre los patrones emocionales se analizaron las tablas de promedio de cada emoción en cada clúster y los mapas de clústeres (ver Apéndice). Tanto en el dendrograma como en los mapas de clústeres puede verse que hay tres grupos de estudiantes que se mantienen estables cuando se consideran 4, 5 o 6 clústeres (Grupos A, B y C, tabla III) y un cuarto grupo que se va disgregando a medida que se aumenta el número de clústeres.

Tabla III. Grupos de estudiantes que se mantienen formando 4, 5 o 6 clústeres

\begin{tabular}{lcll}
\hline & $\begin{array}{c}\text { Número de } \\
\text { estudiantes }\end{array}$ & Emoción destacada & Códigos de los estudiantes \\
\hline Grupo A & 11 & En blanco & $1,6,2,11,9,12,16,3,4,20$ y 25 \\
Grupo B & 2 & Aburrido & 22 y 28 \\
Grupo C & 4 & Feliz & $7,23,8$ y 27 \\
\hline
\end{tabular}

Las tablas de promedios muestran que los tres grupos que se mantienen estables están por encima del promedio en uno de los valores de la variable Emoción y por debajo en el resto (ver Apéndice). Así, el Grupo A está formado por 11 estudiantes que, en promedio, no indicaron ninguna emoción en el 63\% de las actividades; el Grupo B lo componen dos estudiantes que, en promedio, en el $98 \%$ de las actividades declararon sentirse aburridos y en el Grupo $C$ hay 4 estudiantes que, en promedio, en el $84 \%$ de las actividades señalaron que se sentían felices (ver figura 3).

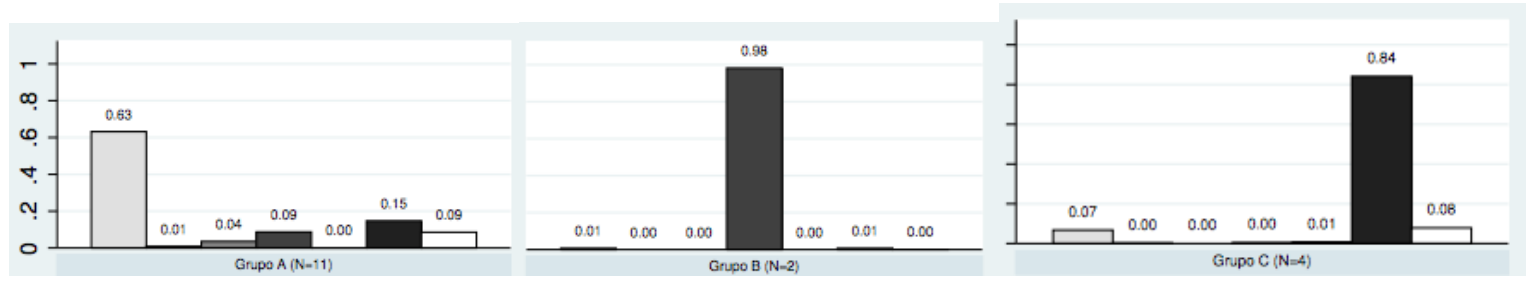

Figura 3. Promedio de cada emoción para los grupos A, B y $C^{1}$

En la figura 4 se observa cómo se va disgregando el grupo formado por los restantes 11 estudiantes. Al pasar de 4 a 5 clústeres, este grupo queda dividido en dos, uno de los cuales está formado por dos estudiantes con promedio muy por encima del promedio total en la emoción Desafiado, se trata de dos alumnos que declararon sentirse desafiados en el 54\% de las actividades, en promedio. Estos dos estudiantes también se encuentran por encima del promedio en la emoción Preocupación y por debajo del promedio en el resto de las emociones y en las respuestas en blanco (ver Apéndice). La división en 6

\footnotetext{
${ }^{1}$ El orden en que están representadas las emociones en el diagrama de barras es: En Blanco, Enfadado, Preocupado, Aburrido, Indiferente, Feliz y Desafiado.
} 
grupos deja estable el grupo con promedio alto en la emoción Desafiado y divide al otro en dos nuevos grupos que son muy similares entre ellos, en cuanto al promedio en cada una de las emociones y similares también al grupo que lo originó.

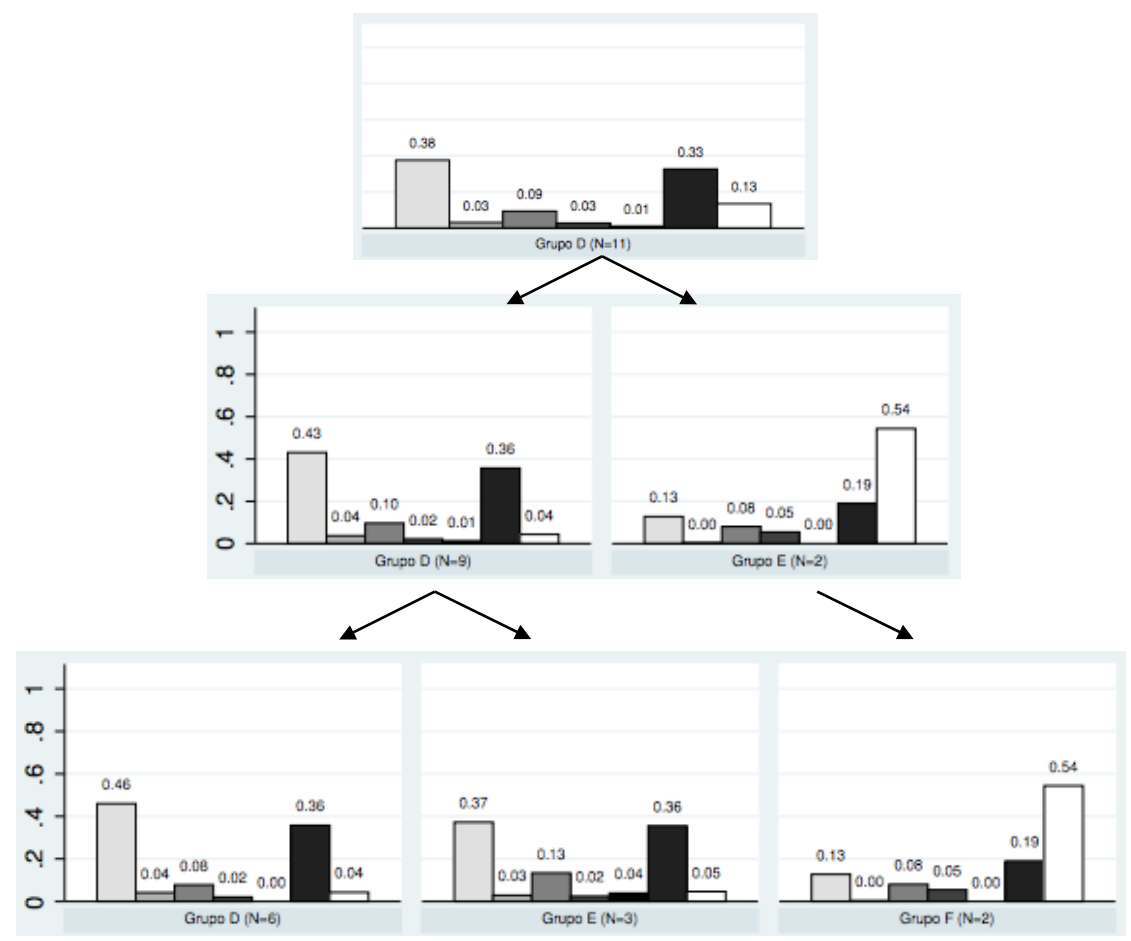

Figura 4. División de un grupo de estudiantes al aumentar el número de clústeres

A partir de este análisis, se consideraron 5 patrones emocionales en el conjunto de los 28 estudiantes, cuyas características principales se resumen en la tabla IV.

Tabla IV. Perfiles emocionales identificados en los estudiantes de $4^{\circ}$ Básico

\begin{tabular}{llc}
\hline Patrón & Descripción & $\begin{array}{c}N^{0} \text { de } \\
\text { estudiantes }\end{array}$ \\
\hline En Blanco & $\begin{array}{l}\text { Estudiantes con un porcentaje alto de actividades donde no indican } \\
\text { ninguna emoción (más del 60\%) y por debajo del promedio en el } \\
\text { resto de las emociones. }\end{array}$ & 11 \\
\hline Feliz & $\begin{array}{l}\text { Estudiantes con un porcentaje alto de actividades en las que } \\
\text { declaran la emoción Feliz (más del 80\%) y por debajo del promedio } \\
\text { en el resto de las emociones. }\end{array}$ & 4 \\
Aburrido & $\begin{array}{l}\text { Estudiantes con un porcentaje alto de actividades en las que } \\
\text { declaran la emoción Aburrido (más del 90\%) y por debajo del } \\
\text { promedio en el resto de las emociones. } \\
\text { Destudiantes con un porcentaje alto de actividades en las que }\end{array}$ & 2 \\
& $\begin{array}{l}\text { Estaran la emoción Desafiado (más del 50\%), por encima del } \\
\text { declamedio en la emoción Preocupadoy por debajo del promedio en el } \\
\text { prosto de las emociones. }\end{array}$ & 2 \\
Pluriemocional & $\begin{array}{l}\text { Estudiantes con el mayor promedio en las emociones Enfadado, } \\
\text { Preocupado e Indiferentey con porcentajes medios y similares en las } \\
\text { actividades donde no declaran emoción (más del 40\%) o declaran } \\
\text { Feliz(36\%). }\end{array}$ & 9 \\
\hline
\end{tabular}




\section{Discusión y conclusiones}

La mayoría de los estudios sobre las emociones de los estudiantes y las Matemáticas se han centrado en la resolución de problemas o en instancias académicas generales como realizar las tareas en casa, responder a una prueba o estudiar (Frenzel et al., 2007a; Martínez-Sierra y García, 2014; Op’t Eynde et al., 2006). El principal aporte de esta investigación es que se estudian las emociones desde un punto de vista local (Goldin, 2000), con foco en las actividades que el docente propone para realizar en cada clase, durante el estudio de una unidad de contenido concreta, en este caso "Medición: unidades de longitud, perímetro y área". El carácter exploratorio de esta investigación limita el tipo de conclusiones que se puede extraer, sin embargo, se ha obtenido información sobre la que sería interesante profundizar.

Retomando las preguntas de investigación, los participantes muestran un mapa emocional con un alto porcentaje de respuestas en blanco (40.6\%) y Feliz (30.7\%), seguido de Aburrido (11.6\%) y Desafiado (9.8\%). El resto de las opciones tuvieron porcentajes bajos: Preocupado (5.1\%), Enfadado (1.6\%) e Indiferente (0.7\%). Estos resultados difieren de los obtenidos en otras investigaciones, donde la emoción que se asocia con mayor frecuencia a las clases y tareas matemáticas es el aburrimiento (Martínez-Sierra y García, 2014), lo que avalaría la necesidad de distinguir entre afectos locales y globales propuesta por Goldin (2000).

Por otra parte, los resultados mostraron que existe relación entre el género de los estudiantes y las emociones que indican, $\chi^{2}(6, N=3836)=124.96, p<0.001$. Se encontraron diferencias significativas en las respuestas en blanco y las opciones Enfadado, Preocupado y Feliz. El porcentaje de veces que se eligió Enfadado (1.6\%) no permite interpretar este resultado para esta emoción. En el resto, los hombres presentaron mayor promedio de respuestas en blanco que las mujeres ( $M=0.44, S D=0.29$ y $M=0.39$, $\mathrm{SD}=0.24$, respectivamente) y se mostraron menos preocupados ( $M=0.01, S D=0.03$ y $M=0.07, S D=0.09$, respectivamente). En la opción Feliz, los promedios son iguales $(M=0.31)$, con SD=0.24 y SD=0.28 en el caso de las mujeres y los hombres. Esta información complementa los resultados de investigaciones realizadas en Alemania y Finlandia (Frenzel et al., 2007a; Holm et al., 2016), donde las mujeres mostraron mayor ansiedad, desesperanza y vergüenza que los hombres y un menor disfrute de la disciplina.

Por último, definir un perfil emocional para cada estudiante y usar métodos de clusterización permitió identificar 5 patrones entre los 28 participantes: En Blanco, Feliz, Aburrido, Desafiado y Pluriemocional. El nombre de los 4 primeros patrones hace referencia a la emoción que sus integrantes indicaron en más de un 50\% de las actividades. En el grupo denominado Pluriemocional están los estudiantes que presentaron mayores promedios en los estados Enfadado, Preocupado e Indiferente.

El método utilizado permitió obtener información desde el punto de vista de los propios estudiantes y directamente en el momento de realizar cada actividad, dando a los resultados el carácter de situados en contexto (Volet y Järvelä, 2001). Sin embargo, ese método también aporta ciertas limitaciones: una de ellas dada por el uso de un listado concreto de 6 emociones que puede haber influido en el alto porcentaje de respuestas en blanco, ya que los estudiantes podrían no haber identificado en esas opciones lo que estaban sintiendo al realizar la actividad. Por otra parte, el conjunto de actividades donde los participantes indicaron sus emociones corresponde a diferentes niveles cognitivos, desde conocer una definición, aplicar una fórmula o resolver problemas planteados en un contexto matemático o no matemático. Este es un elemento que puede haber influido en lo que sentían los estudiantes y que no ha sido considerado en esta investigación. Otros factores que podrían influir en el dominio emocional están relacionados con la gestión que se realice de las actividades, por ejemplo, que sean individuales o grupales, el tipo de interacción que se dé con el profesor y entre los estudiantes o los materiales que se utilicen.

Finalmente, la distancia Hamming (1950) permitió observar la variedad de experiencias emocionales que se suceden en el día a día de las clases de Matemáticas y, con los métodos de clusterización, identificar cinco patrones emocionales, de los que surgen diversas preguntas: ¿Se mantendrán esos perfiles y patrones en las actividades de otra unidad de contenidos, por ejemplo, de números o de datos y azar?, ¿y en niveles educativos superiores? 
Esta investigación complementa los resultados de investigaciones previas sobre las emociones académicas, en particular sobre las emociones relacionadas con las actividades (Pekrun et al., 2011) y abre nuevas interrogantes. Los resultados muestran la necesidad de ampliar los conocimientos acerca del dominio emocional y sus características en el contexto diario. La realización de cualquier actividad está ligada a una emoción, es lo que podríamos llamar emociones locales; si esas emociones locales se repiten, darán lugar a emociones globales o estados emocionales más estables en el tiempo y difícilmente modificables (Goldin, 2000). Es importante generar un mayor conocimiento de esas emociones locales ya que, como señalaba Mandler (como se cita en McLeod, 1992, p. 578) las emociones son la base sobre la que se van construyendo las actitudes y creencias hacia la disciplina.

\section{Referencias}

Atlas, R., y Overall, J. (1994). Comparative evaluation of two superior stopping rules for hierarchical cluster analysis. Psychometrika, 59(4), 581-591.

Buehl, M. M. y Alexander, P. A. (2005). Motivation and performance differences in students' domainspecific epistemological belief profiles. American Educational Research Journal, 42(4), 697-726.

DeBellis, V. A. y Goldin, G. A. (2006). Affect and meta-affect in mathematical problem solving: a representational perspective. Educational Studies in Mathematics, 63, 131-147.

Frenzel, A. C., Pekrun, R. y Goetz, T. (2007a). Girls and mathematics - A "hopeless" issue? A control-value approach to gender differences in emotions towards mathematics. European Journal of Psychology of Education, XXII(4), 497-514.

Frenzel, A. C., Pekrun, R. y Goetz, T. (2007b). Perceived learning environment and students' emotional experiences: A multilevel analysis of mathematics classrooms. Learning and Instruction, 17, 478-493.

Goetz, T., Frenzel, A. C., Hall, N. C. y Pekrun, R. (2008). Antecedents of academic emotions: Testing the internal/external frame of reference model for academic enjoyment. Contemporary Educational Psychology, 33, 9-33.

Goldin, G. A. (2000). Affective pathways and representation in mathematical problem solving. Mathematical Thinking and Learning, 2(3), 209-219.

Hamming, R. (1950). Error detecting and error correcting codes. Bell System Technical Journal, 29(2), 147160.

Hannula, M. (2012). Exploring new dimensions of mathematics-related affect: embodied and social theories. Research in Mathematics Education, 14(2), 137-161.

Hannula, M. S., Pantziara, M., Waege, K. y Scholöglmann, W. (2010). Introduction multimethod approaches to the multidimensional affect in mathematics education. En V. Durand-Guerrier, S. Soury-Lavergne, y F. Arzarello (Eds.), Proceedings of the Sixth Congress of the European Society for Research in Mathematics Education, 28-33. Lyon: Service des publications.

Holm, M. E., Hannula, M. S. y Björn, P. M. (2016). Mathematics-related emotions among Finish adolescents across different performance levels. Educational Psychology, 37, (2). doi:10.1080/01443410.2016.1152354

Ibáñez, N. (2002). Las emociones en el aula. Estudios Pedagógicos, 28, 31-45.

McLeod, D. B. (1992). Research on affect in mathematics education: a reconceptualization. En D. A. Grouws (Ed.). Handbook of research on mathematics teaching and learning. A project of the national council of teachers of mathematics, 23, 575-596. Nueva York: MacMillan. 
Mandler, G. (1989). Affect and learning: Causes and Consequences of Emotional Interactions. En D. B. McLeod y V. M. Adams (Eds.), Affect and mathematical problem solving: a new perspective (3-19). Nueva York: Springer-Verlag.

Martínez-Sierra, G. y García, M. S. (2014). High school students' emotional experiences in mathematics classes. Research in Mathematics Education, 16(3), 234-250. doi:10.1080/14794802.2014.895676

Op't Eynde, P., De Corte, E. y Verschaffel, L. (2006). Accepting emotional complexity: A socioconstructivist perspective on the role of emotions in the mathematics classroom. Educational Studies in Mathematics, 63, 193-207.

Pekrun, R. (2005). Progress and open problems in educational emotion research. Learning and Instruction, 15, 497-506.

Pekrun, R., Goetz, T., Frenzel, A. C., Barchfeld, P. y Perry, R. P. (2011). Measuring emotions in students' learning and performance: The Achievement Emotions Questionnaire (AEQ). Contemporary Educational Psychology, 36, 36-48.

Pekrun, R., Goetz, T., Titz, W. y Perry, R. P. (2002). Academic emotions in students' self-regulated learning and achievement: A program of qualitative and quantitative research. Educational Psychologist, 37, 91106.

Schoenfeld, A. (1998). Toward a theory of teaching-in-context. Issues in Education, 4(1), 1-94.

Volet, S. y Järvelä, S. (Eds.). (2001). Motivation in learning contexts: Theoretical advances and methodological implications. Reino Unido: Elsevier. 


\section{Apéndice}

Tablas de promedios y mapas de los clústeres 4,5 y 6, respectivamente.

\begin{tabular}{|l|c|c|c|c|c|c|c|c|}
\hline & EnBlanco & Enojo & Preocupación & Aburrido & Indiferente & Alegre & Desafiado \\
\hline Grupo A-(N=11) & 0.6317 & 0.0093 & 0.0358 & 0.0869 & 0.0033 & 0.1473 & 0.0856 \\
\hline Grupo B-(N=2) & 0.0073 & 0.0000 & 0.0000 & 0.9818 & 0.0000 & 0.0073 & 0.0036 \\
\hline Grupo C-(N=4) & 0.0675 & 0.0018 & 0.0000 & 0.0036 & 0.0073 & 0.8412 & 0.0785 \\
\hline Grupo D-(N=11) & 0.3756 & 0.0312 & 0.0936 & 0.0279 & 0.0106 & 0.3265 & 0.1347 \\
\hline Promedio & 0.4059 & 0.0162 & 0.0508 & 0.1157 & 0.0065 & 0.3068 & 0.0980 \\
\hline 10
\end{tabular}

\begin{tabular}{|l|c|c|c|c|c|c|c|}
\hline & EnBlanco & Enojo & Preocupación & Aburrido & Indiferente & Alegre & Desafiado \\
\hline Grupo A-(N=11) & 0.6317 & 0.0093 & 0.0358 & 0.0869 & 0.0033 & 0.1473 & 0.0856 \\
\hline Grupo B-(N=2) & 0.0073 & 0.0000 & 0.0000 & 0.9818 & 0.0000 & 0.0073 & 0.0036 \\
\hline Grupo C-(N=4) & 0.0675 & 0.0018 & 0.0000 & 0.0036 & 0.0073 & 0.8412 & 0.0785 \\
\hline Grupo D-(N=9) & 0.4307 & 0.0373 & 0.0965 & 0.0219 & 0.0130 & 0.3569 & 0.0438 \\
\hline Grupo E-(N=2) & 0.1277 & 0.0036 & 0.0803 & 0.0547 & 0.0000 & 0.1898 & 0.5438 \\
\hline Promedio & 0.4059 & 0.0162 & 0.0508 & 0.1157 & 0.0065 & 0.3068 & 0.0980 \\
\hline
\end{tabular}

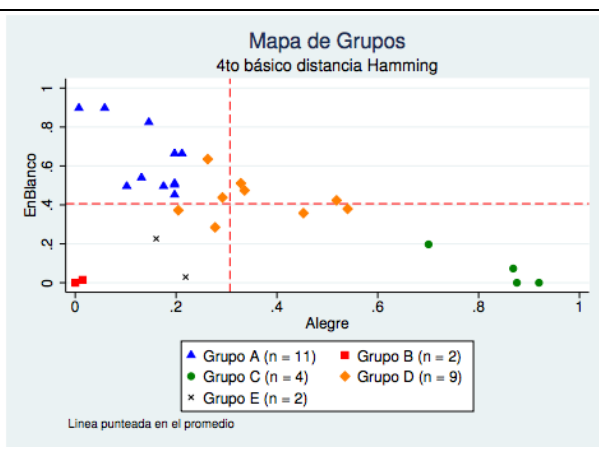

\begin{tabular}{|l|c|c|c|c|c|c|c|}
\hline & EnBlanco & Enojo & Preocupación & Aburrido & Indiferente & Alegre & Desafiado \\
\hline Grupo A-(N=11) & 0.6317 & 0.0093 & 0.0358 & 0.0869 & 0.0033 & 0.1473 & 0.0856 \\
\hline Grupo B-(N=2) & 0.0073 & 0.0000 & 0.0000 & 0.9818 & 0.0000 & 0.0073 & 0.0036 \\
\hline Grupo C-(N=4) & 0.0675 & 0.0018 & 0.0000 & 0.0036 & 0.0073 & 0.8412 & 0.0785 \\
\hline Grupo D-(N=6) & 0.4599 & 0.0414 & 0.0779 & 0.0207 & 0.0000 & 0.3577 & 0.0426 \\
\hline Grupo E-(N=3) & 0.3723 & 0.0292 & 0.1338 & 0.0243 & 0.0389 & 0.3552 & 0.0462 \\
\hline Grupo F-(N=2) & 0.1277 & 0.0036 & 0.0803 & 0.0547 & 0.0000 & 0.1898 & 0.5438 \\
\hline Promedio & 0.4059 & 0.0162 & 0.0508 & 0.1157 & 0.0065 & 0.3068 & 0.0980 \\
\hline
\end{tabular}

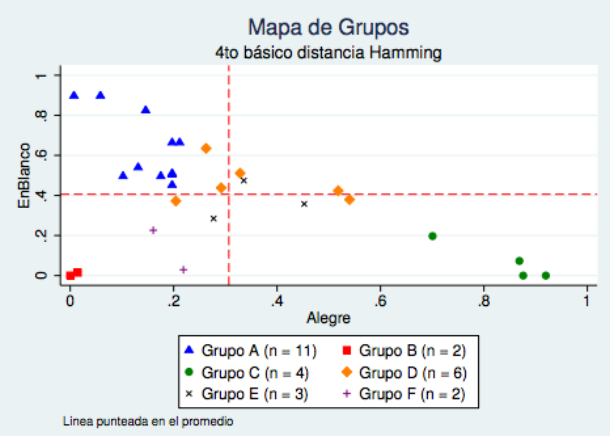

